\title{
Fast ISAR imaging method based on scene segmentation
}

\author{
Mingjiu Lü*, Shaodong Li, Wenfeng Chen, Jun Yang, and Xiaoyan Ma
}

Air Force Early Warning Academy, Wuhan 430019, China

\begin{abstract}
Although compressed sensing inverse synthetic aperture radar (ISAR) imaging methods are widely used in radar signal processing, its reconstructing time and memory storage space requirements are very high. The main reason is that large scene reconstruction needs a higher dimension of the sensing matrix. To reduce this limitation, a fast high resolution ISAR imaging method, which is based on scene segmentation for random chirp frequencystepped signals, is proposed. The idea of scene segmentation is used to solve the problems aforementioned. In the method, firstly, the observed scene is divided into multiple sub-scenes and then the sub-scenes are reconstructed respectively. Secondly, the whole image scene can be obtained through the stitching of the sub-scenes. Due to the reduction of the dimension of the sensing matrix, the requirement of the memory storage space is reduced substantially. In addition, due to the nonlinear superposition of the reconstructed time of the segmented sub-scenes, the reconstruction time is reduced, and the purpose of fast imaging is achieved. Meanwhile, the feasibility and the related factors which affect the performance of the proposed method are also analyzed, and the selection criterion of the scene segmentation is afforded. Finally, theoretical analysis and simulation results demonstrate the feasibility and effectiveness of the proposed method.
\end{abstract}

Keywords: compressed sensing (CS), inverse synthetic aperture radar (ISAR) imaging, random chirp frequency-stepped signal, scene segmentation.

DOI: $10.21629 / J S E E .2017 .06 .06$

\section{Introduction}

Nowadays, inverse synthetic aperture radar (ISAR) is widely applied in many civilian and military fields. It achieves the high range resolution by transmitting a large bandwidth signal, and the high cross-range resolution is obtained from the diversity of the radar visual angle to the target. For the modern ISAR applications, high image resolution is usually essential to extract target features. However, with the requirement of image resolution increasing, the high sampling rate and the high data rate have brought

\footnotetext{
Manuscript received November 22, 2016

*Corresponding author.

This work was supported by the National Natural Science Foundation of China (61671469).
}

many difficulties to radar system design and processing. Compressed sensing (CS) [1] provides a new solution to the aforementioned shortcomings in recent years $[2,3]$. Then the system complexity and the data amount can be reduced significantly by emitting the random chirp frequency-stepped signal (RCFS), which transfers the randomness of CS from the receiver to the transmitter [4,5], while the anti-interference performance is enhanced, thus this waveform has attracted widespread attention [6-8].

However, the main problems of ISAR RCFS imaging methods based on CS are the long-time reconstruction and the high requirements of memory storage space $[6,9]$. For example, assuming the observed scene is a $512 \times 512$ matrix and the dimension of the measurement data matrix is $128 \times 128$, therefore the dimension of the sensing matrix will reach $16384 \times 262144$. To deal with such a large matrix, the processing time and required memory storage space should be large enough as well. The existing researches mainly focus on exploring new imaging algorithms and imaging models to overcome these problems. From the view of the algorithm, it is mainly to explore the optimization algorithm which has higher convergence speed, e.g. the matching pursuit algorithms [10] and the SL0 algorithms [11]. From the perspective of imaging models, they can be generally classified into two categories: one kind is the two-dimensional joint model, and the data are processed directly in the form of matrix [12-15]. However, this method requires that the transmitted signal form must be the same, so the applicable scope of this model is limited. The other is to transform the two-dimensional joint model into two-dimensional decoupling model (i.e. range direction imaging first, and then cross-range direction imaging). However, when the resolution is high or the observed scene is large, this method still needs a long reconstruction time and the data storage requirement is also growing. Besides, a range segmented reconstruction strategy for the CS SAR imaging scheme was proposed in [16], but still relied heavily on computer performance.

To overcome the above-mentioned problems, the idea 
of scene segmentation is used to divide the observed scene into several sub-scenes in this paper. Firstly, based on the characteristics of the weak correlation between the echo data of the sub-scenes, the problem of large scene reconstruction is exchanged into the problem of several subscenes reconstruction. Secondly, the corresponding sparse reconstruction models are established for each sub-scene, and the sub-scenes are reconstructed respectively. Finally, all the sub-scenes are stitched together to get the whole image. By segmenting the observed scene, the scattered points are divided into different sub-scenes, so that the number of scattered points which need to be reconstructed is reduced in several sub-scenes, so the dimension of the required sensing matrix is decreased. Then the purpose of diminishing the requirement of the storage space is achieved. Due to the nonlinear superposition of the reconstructed computation time, the total processing time is also lessened. In addition, the factors which affect the performance of the proposed method are analyzed, and the principle of scene segmentation is presented. At the same time, using the idea of smooth treatment, the reconstruction error caused by the discontinuity of the data in the sub-scene boundary is dwindled. Because all the sub-scenes are independent of each other, the sub-scene reconstruction can be processed in parallel, and the computation time can be further shortened. The proposed method has a high reference and application value in the field of real-time ISAR imaging.

\section{Echo model of RCFS ISAR based on CS}

The RCFS signal can be considered as a random emission form of the traditional chirp frequency stepped-signal (CFS). Assuming that a burst of CFS signals contains $N$ sub-pulses, although only $M(M<N)$ sub-pulses are randomly emitted from the RCFS signal, the equivalent bandwidth can be achieved as well [6,7]. In the following section, the RCFS ISAR imaging model is analyzed.

The waveform of the RCFS can be denoted as

$$
\begin{gathered}
s_{r}\left(\widehat{t}, m, n_{a}\right)=\operatorname{rect}\left[\frac{\widehat{t}}{T}\right] \exp \left[\mathrm{j} \pi \mu \widehat{t}^{2}\right] . \\
\exp \left[\mathrm{j} 2 \pi\left(f_{0}+m_{n_{a}} \Delta f\right) \widehat{t}\right]
\end{gathered}
$$

where $\operatorname{rect}(\cdot)$ denotes a rectangular window, $M$ is the number of sub-pulses in a burst, $n_{a}=0,1,2, \ldots, N a$ denotes the $n_{a}$ th burst, $N a$ is the number of bursts, $\widehat{t}$ is the fast time satisfying $\widehat{t}=\left[-T / 2,-T / 2+1 / f_{s}, \ldots,-T / 2+\right.$ $\left.(H-1) / f_{s}\right]$ in which $T$ is the pulse width, $f_{s}$ is the sampling frequency, $B$ is the sub-pulse bandwidth, $\mu=B / T$ is the chirp rate, $\Delta f$ is the frequency step, $m_{n_{a}}$ is a subset of $[0: N-1]$, and $m$ runs 1 to $M$.
For the scattered points at range $R_{k}$, neglecting the reflection coefficient, the echo after Dechirp [17] can be expressed as

$$
\begin{aligned}
& S_{f}\left(f, m, n_{a}\right)=\sum_{k=0}^{K-1} \sigma_{k} \cdot \operatorname{sinc}\left[T\left(f+2 \mu \Delta R_{k} / c\right)\right] . \\
& \exp \left\{-\mathrm{j}\left[\frac{4 \pi}{c} f_{m_{n_{a}}} \Delta R_{k}\right]\right\}
\end{aligned}
$$

where $f_{m_{n_{a}}}=f_{0}+m_{n_{a}} \Delta f, \sigma_{k}$ denotes intensity of the $k$ th scattered point, and $K$ is the number of the scattered points.

Supposing that the target motion compensation has been completed, and then $\Delta R_{k}$ [2] can be approximated as

$$
\Delta R_{k} \approx y_{k}+x_{k} \omega t_{a}
$$

where $\omega$ is the rotational angular velocity, $\left(x_{k}, y_{k}\right)$ is the location of the $k$ th scattered point in the target coordinates, and $t_{a}$ is the slow time satisfying $t_{a}=\left(n_{a}-1\right) M T_{r}$ in which $T_{r}$ is the pulse repetition interval (PRI).

Transforming (2) into time domain yields

$$
\begin{gathered}
s\left(\widehat{t}, m, n_{a}\right)=\sum_{k=0}^{K-1} \sigma_{k} \operatorname{rect}\left(\frac{\widehat{t}}{T}\right) . \\
\exp \left\{-\mathrm{j}\left[\frac{4 \pi}{c}\left(f_{m_{n_{a}}}+\mu \widehat{t}\right) \Delta R_{k}\right]\right\} .
\end{gathered}
$$

An ISAR image reflects the locations and intensions of the strong points of the target. Strong scattered points usually take up only a fraction of the observed scene. However, echoes from weak scattered points contribute little to the image formation. Therefore, the scattered points are expected to be sparse in the ISAR image and ISAR imaging can be formulated as a sparse recovery problem. When building the sparse reconstruction model of the observed scene, echoes are usually turned into vector forms, which leads to the higher requirement of the processing capacity of the processing equipment and memory storage space, or even impossibility of achieving [6,7]. In order to alleviate these problems, this paper builds the sparse reconstruction model based on the two-dimensional decoupling model. First, the range sparse reconstruction model can be expressed as

$$
\boldsymbol{S}\left(n_{a}\right)=\boldsymbol{\Psi}^{\prime}\left(n_{a}\right) \boldsymbol{\theta}\left(n_{a}\right)+\boldsymbol{E}
$$

where $\boldsymbol{S}\left(n_{a}\right)$ denotes the echo signal of the $n_{a}$ th burst, $(\cdot)^{\mathrm{T}}$ is the transpose operator, and $\boldsymbol{S}\left(n_{a}\right)=$ $\left[s\left(\widehat{t}, 1, n_{a}\right), \ldots, s\left(\widehat{t}, M, n_{a}\right)\right]^{\mathrm{T}} \in \mathbf{C}^{M H \times 1}, \boldsymbol{\theta}\left(n_{a}\right) \in$ $\mathbf{C}^{P \times 1}$ is the range profile, and $P$ is the sampling points of the range, and $\boldsymbol{E}$ is the additive noise. $\boldsymbol{S}\left(n_{a}\right)$ can be regarded as the measurement data of the traditional CFS signal because only $M$ sub-pulses are transmitted, $\boldsymbol{\Psi}^{\prime}\left(n_{a}\right)=$ $\left[\boldsymbol{\Psi}^{\prime}\left(n_{a}, 1\right), \ldots, \boldsymbol{\Psi}^{\prime}\left(n_{a}, m\right), \ldots, \boldsymbol{\Psi}^{\prime}\left(n_{a}, M\right)\right]^{\mathrm{T}}$ is the sen- 
sing matrix, and $\boldsymbol{\Psi}^{\prime}\left(n_{a}, m\right) \in \mathbf{C}^{H \times P}$ satisfying $\Psi^{\prime}\left(n_{a}, m\right)=\exp \left[-\mathrm{j} 4 \pi\left(f_{m_{n_{a}}}+\mu \widehat{t}(h)\right) \Delta R(p) / c\right]$ in which $\Delta R$ is the discrete length of the range.

For $N_{a}$ bursts, we can obtain

$$
\boldsymbol{S}=\boldsymbol{\Psi}^{\prime} \theta+\boldsymbol{E}
$$

where $\boldsymbol{S}=[\boldsymbol{S}(1), \boldsymbol{S}(2), \ldots, \boldsymbol{S}(N a)] \in \mathbf{C}^{M H \times N a}, \boldsymbol{\Psi}^{\prime}$ is an $M H \times P N a$ matrix, and $\boldsymbol{\theta}=[\boldsymbol{\theta}(1), \boldsymbol{\theta}(2), \ldots, \boldsymbol{\theta}(N a)] \in$ $\mathbf{C}^{P \times N a}$.

For the cross-range direction, the reconstruction model can be written as

$$
\boldsymbol{Y}^{\prime}=\boldsymbol{\Phi}^{\prime} \boldsymbol{\theta}^{\mathrm{T}}=\boldsymbol{\Phi} \boldsymbol{H}^{\prime} \boldsymbol{\sigma}^{\mathrm{T}}+\boldsymbol{E}=\boldsymbol{\Psi}_{a}^{\prime} \boldsymbol{\sigma}^{\mathrm{T}}+\boldsymbol{E}
$$

where $\boldsymbol{Y}^{\prime} \in \mathbf{C}^{N a^{\prime} \times P}, N a^{\prime}<N a$ is the randomly selected cross-range data, $\boldsymbol{\Phi}^{\prime} \in \mathbf{R}^{N a^{\prime} \times N a}$ is the measurement matrix, $\boldsymbol{\Psi}_{a}^{\prime}=\left[\boldsymbol{\Psi}_{a}^{\prime}(1), \ldots, \boldsymbol{\Psi}_{a}^{\prime}\left(n_{a}\right), \ldots, \boldsymbol{\Psi}_{a}^{\prime}(N a)\right]$ is the sensing matrix, and $\boldsymbol{\Psi}_{a}^{\prime}\left(n_{a}\right) \in \mathbf{R}^{N a^{\prime} \times Q}$, $\boldsymbol{H}^{\prime} \in \mathbf{C}^{N a \times Q}$ is the basis matrix satisfying $\boldsymbol{H}^{\prime}=$ $\exp \left[-\mathrm{j} 4 \pi f_{0} \omega \Delta L(q)\left(n_{a}-1\right) M T_{r} / c\right]$ in which $\Delta L$ is the discrete length of the cross-range, and $\boldsymbol{\sigma} \in \mathbf{C}^{P \times Q}$ is the observed scene.

So far, the reconstruction model of RCFS ISAR based on CS is obtained by (6) and (7). From (6) and (7), we can get the size of $\sigma$, that is the sampling points of the observed scene, and directly impacts the size of the sensing matrix $\boldsymbol{\Psi}^{\prime}$ and $\boldsymbol{\Psi}_{a}^{\prime}$. In order to reduce the reconstruction time and the memory storage space requirement, the sampling points or the observed scene size need to decrease, which we would rather not see. Therefore, a fast imaging method is studied in the next section.

\section{Fast imaging method based on scene segmentation}

When using CS to reconstruct the observed scene, there are two main factors that determine the processing time: one is the number of scattered points in the scene; the other is the dimension of the sensing matrix, which also determines the demand of memory storage space. Based on the above reasons, the original scene is divided into several sub-scenes in this paper, and due to the nonlinear superposition of the reconstructed time, the reconstruction time is shortened. On the other hand, the dimension of the sensing matrix is reduced which makes the memory storage space requirement lower. First of all, the principle of reducing the reconstruction time is given by the method of scene segmentation.

Suppose $A$ represents the number of scatter points in the observed scene, $B$ and $C$ are the dimensions of the sensing matrix. The range and cross-range of the observed scene are divided into $I$ and $J$ segments respectively, that is, the whole scene is divided into $D=I J$ sub-scenes. For the sub-scene, the range points are $P^{\prime}=P / I$ and the cross-range points are $Q^{\prime}=Q / J$. Then the computational complexity can be expressed as $O(A B C)$ before the scene segmentation and $O(A B C / D I J)$ is the computational complexity after the scene segmentation. Apparently, the following formula is satisfied:

$$
O(A B C) \leqslant D\left[O\left(\frac{A}{D} \frac{B}{I} \frac{C}{J}\right)\right]=D\left[O\left(\frac{A B C}{D^{2}}\right)\right] .
$$

Clearly, after the scene segmentation, the scattered points are divided into sub-scenes, and the corresponding sensing matrix dimension is reduced, which can reduce the reconstruction time and the memory storage space requirement.

Fig. 1 shows the specific implementation process of the scene segmentation and the main steps of the method are as follows:

Step 1 We first divide the scene in the range direction, and the range imaging reconstruction model is constructed to obtain the corresponding range imaging of sub-scenes.

Step 2 Divide the observed scene in the cross-range direction, and the corresponding cross-range imaging reconstruction model is constructed. Then the sub-scene reconstruction is accomplished.

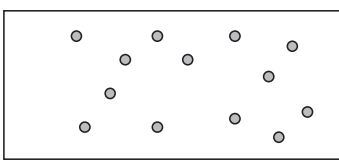

Observed scene



Stitched scene
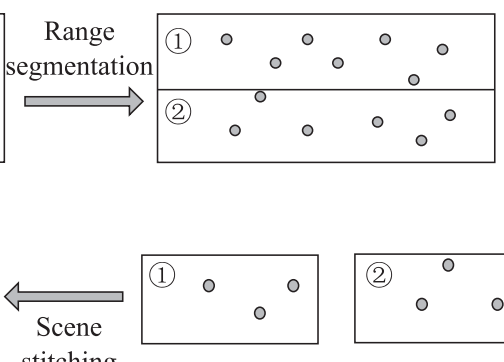
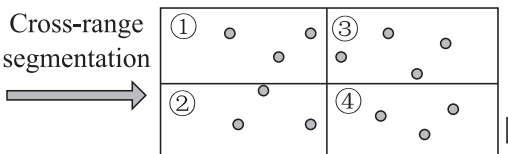

Sub-scene reconstrction
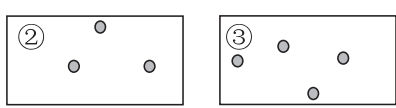

Fig. 1 Sketch of the scene segmentation method 
Step 3 Stitch all the sub-scenes according to the segmentation order, and finally obtain the whole image.

Because the reconstruction of each sub-scene is independent, the parallel processing method can be adopted, and the computation time will be further reduced.

Based on the above analysis, the detailed implementation process of the fast imaging method based on the idea of scene segmentation is given in the following section.

(i) Range direction reconstruction

Firstly, the range reconstruction of the sub-scene is performed. Segmenting the scene along the range direction and we can obtain $I$ sub-scenes. Then the reconstruction processing can be written as

$$
\left\{\begin{array}{c}
\boldsymbol{S}=\boldsymbol{\Psi}_{1} \boldsymbol{\theta}_{1}+\boldsymbol{E}_{1} \\
\vdots \\
\boldsymbol{S}=\boldsymbol{\Psi}_{i} \boldsymbol{\theta}_{i}+\boldsymbol{E}_{i} \\
\vdots \\
\boldsymbol{S}=\boldsymbol{\Psi}_{I} \boldsymbol{\theta}_{I}+\boldsymbol{E}_{I}
\end{array}\right.
$$

where $\boldsymbol{\theta}_{i}=\left[\boldsymbol{\theta}_{i}(1), \boldsymbol{\theta}_{i}(2), \ldots, \boldsymbol{\theta}_{i}(N a)\right]$ is the range profile of the $i$ th sub-scene, $\boldsymbol{\theta}_{i}=\left[\boldsymbol{\theta}_{i}(1), \boldsymbol{\theta}_{i}(2), \ldots, \boldsymbol{\theta}_{i}(N a)\right]$ is a $P^{\prime} \times N a$ matrix, $P^{\prime}=P / I, \quad \Psi_{i}=$ $\left[\boldsymbol{\Psi}_{i}(1), \boldsymbol{\Psi}_{i}(2), \ldots, \boldsymbol{\Psi}_{i}(N a)\right]$ is the sensing matrix and the component $\boldsymbol{\Psi}_{i}\left(n_{a}\right)$ satisfying $\boldsymbol{\Psi}_{i}\left(n_{a}\right)=$ $\left[\boldsymbol{\Psi}_{i}\left(n_{a}, 1\right), \ldots, \boldsymbol{\Psi}_{i}\left(n_{a}, M\right)\right]^{\mathrm{T}}$ in which $\boldsymbol{\Psi}_{i}\left(n_{a}, m\right)$ is an $H \times P^{\prime}$ matrix, $\boldsymbol{\Psi}_{i}\left(n_{a}, m\right)$ can be expressed as

$$
\boldsymbol{\Psi}_{i}\left(n_{a}, m\right)=\left[\begin{array}{ccccc}
\exp \left[\mathrm{j} a F_{m}(1) \Delta R_{i}(1)\right] & \cdots & \exp \left[\mathrm{j} a F_{m}(1) \Delta R_{i}(p)\right] & \cdots & \exp \left[\mathrm{j} a F_{m}(1) \Delta R_{i}\left(P^{\prime}\right)\right] \\
\vdots & \ddots & \vdots & \ddots & \vdots \\
\exp \left[\mathrm{j} a F_{m}(h) \Delta R_{i}(1)\right] & \cdots & \exp \left[\mathrm{j} a F_{m}(h) \Delta R_{i}(p)\right] & \cdots & \exp \left[\mathrm{j} a F_{m}(h) \Delta R_{i}\left(P^{\prime}\right)\right] \\
\vdots & \ddots & \vdots & \ddots & \vdots \\
\exp \left[\mathrm{j} a F_{m}(H) \Delta R_{i}(1)\right] & \cdots & \exp \left[\mathrm{j} a F_{m}(H) \Delta R_{i}(p)\right] & \cdots & \exp \left[\mathrm{j} a F_{m}(H) \Delta R_{i}\left(P^{\prime}\right)\right]
\end{array}\right]
$$

where $\Delta R_{i}$ denotes the discrete length of the $i$ th sub-scene in the range direction.

According to the theory of electromagnetic detection [18], no matter in what form the radar signal is, the information of the target is mainly reflected in the amplitude and phase of the echo signal. For the RCFS signal, although sub-pulses in each burst is different, but they are reflected by the same target, the echo signals contain the same target information. That is to say, the echo signals have the same sparse feature. Similarly, when the range is segmented, the range profile of each sub-scene also has joint sparse feature. Therefore, the distributed compressed sensing theory [19] can be used to reconstruct (9), which can improve the accuracy of the range profile reconstruction. It can be expressed as

$$
\begin{aligned}
& \widehat{\boldsymbol{\theta}}_{i}=\arg \min \operatorname{Supp}\left(\boldsymbol{\theta}_{i}\right) \\
& \text { s.t. }\left\|\boldsymbol{S}-\boldsymbol{\Psi}_{i} \boldsymbol{\theta}_{i}\right\|_{2}^{2} \leqslant e
\end{aligned}
$$

where $\operatorname{Supp}\left(\boldsymbol{\theta}_{i}\right)=\bigcup_{n_{a}} \operatorname{Supp}\left(\boldsymbol{\theta}_{i}\left(n_{a}\right)\right)$ denotes nonzero line position set, and $e$ is the noise level.

(ii) Cross-range direction reconstruction

By using the difference of Doppler information, the difference of the scattered points in the same resolution cell can be distinguished. In order to reduce the cross range reconstruction time, the cross range direction can be divided into $J$ sub-scenes. Let $A(i, j)$ denote the $(i, j)$ th sub-scene, then the $A(i, j)$ th sub-scene reconstruction process can be written as

$$
\begin{gathered}
\boldsymbol{\theta}_{i}^{\mathrm{T}}=\boldsymbol{H}_{j} \boldsymbol{\sigma}_{i, j}^{\prime \mathrm{T}}+\boldsymbol{E} \\
i=1,2, \ldots, I ; j=1,2, \ldots, J ; n=1,2, \ldots, P^{\prime}
\end{gathered}
$$

where $\boldsymbol{\sigma}_{i, j}^{\prime} \in \mathbf{C}^{P^{\prime} \times Q^{\prime}}$ is the reflection coefficient matrix of the $A(i, j)$ th sub-scene, the sensing matrix $\boldsymbol{H}_{j} \in \mathbf{C}^{N a \times Q^{\prime}}$ can be expressed as

$$
\boldsymbol{H}_{j}=\left[\begin{array}{ccccc}
\exp \left\{\mathrm{j} b \Delta L_{j}(1) T_{a}(1)\right\} & \cdots & \exp \left\{\mathrm{j} b \Delta L_{j}(q) T_{a}(1)\right\} & \cdots & \exp \left\{\mathrm{j} b \Delta L_{j}\left(Q^{\prime}\right) T_{a}(1)\right\} \\
\vdots & \ddots & \vdots & \ddots & \vdots \\
\exp \left\{\mathrm{j} b \Delta L_{j}(1) T_{a}\left(n_{a}\right)\right\} & \cdots & \exp \left\{\mathrm{j} b \Delta L_{j}(q) T_{a}\left(n_{a}\right)\right\} & \cdots & \exp \left\{\mathrm{j} b \Delta L_{j}\left(Q^{\prime}\right) T_{a}\left(n_{a}\right)\right\} \\
\vdots & \ddots & \vdots & \ddots & \vdots \\
\exp \left\{\mathrm{j} b \Delta L_{j}(1) T_{a}(N a)\right\} & \cdots & \exp \left\{\mathrm{j} b \Delta L_{j}(q) T_{a}(N a)\right\} & \cdots & \exp \left\{\mathrm{j} b \Delta L_{j}\left(Q^{\prime}\right) T_{a}(N a)\right\}
\end{array}\right]
$$


where $\Delta L_{j}$ denotes the discrete length of the $j$ th sub-scene in the cross range direction, and $Q^{\prime}=Q / I$.

From a CS theory point of view, $\boldsymbol{Y}_{i}$ can be viewed as a low-dimensional measurement of $\boldsymbol{\theta}_{i}$ :

$$
\begin{gathered}
\boldsymbol{Y}_{i}=\boldsymbol{\Phi} \boldsymbol{\theta}_{i}^{\mathrm{T}}=\boldsymbol{\Phi} \boldsymbol{H}_{j} \boldsymbol{\sigma}_{i, j}^{\prime \mathrm{T}}+\boldsymbol{E}=\boldsymbol{\Theta}_{j} \boldsymbol{\sigma}_{i, j}^{\prime \mathrm{T}}+\boldsymbol{E}, \\
i=1,2, \ldots, I ; j=1,2, \ldots, J
\end{gathered}
$$

where $\boldsymbol{Y}_{i} \in \mathbf{C}^{N a^{\prime} \times P^{\prime}}, N a^{\prime}<N a$ is the measurement data, $\boldsymbol{\Phi} \in \mathbf{R}^{N a^{\prime} \times N a}$ is a measurement matrix, and $\boldsymbol{\Theta}_{j} \in$ $\mathbf{R}^{N a^{\prime} \times Q^{\prime}}$ is a sensing matrix.

Similarly, the final result $\boldsymbol{\sigma}_{i, j}^{\prime}$ can be obtained by solving the following expression:

$$
\begin{gathered}
\min \left\|\boldsymbol{\sigma}_{i, j}^{\prime \mathrm{T}}\right\|_{1} \\
\text { s.t. }\left\|\boldsymbol{Y}_{i}-\boldsymbol{\Theta}_{j} \boldsymbol{\sigma}_{i, j}^{\prime \mathrm{T}}\right\|_{2}^{2} \leqslant e .
\end{gathered}
$$

(iii) Sub-scenes splicing

For $\boldsymbol{\sigma}_{i, j}^{\prime}$, splice sub-scenes according to the segmentation order and obtain the final big scene finally.

$$
\boldsymbol{\sigma}=\left[\begin{array}{ccccc}
\boldsymbol{\sigma}_{1,1}^{\prime} & \cdots & \boldsymbol{\sigma}_{1, j}^{\prime} & \cdots & \boldsymbol{\sigma}_{1, J}^{\prime} \\
\vdots & \ddots & \vdots & \ddots & \vdots \\
\boldsymbol{\sigma}_{i, 1}^{\prime} & \cdots & \boldsymbol{\sigma}_{i, j}^{\prime} & \cdots & \boldsymbol{\sigma}_{i, J}^{\prime} \\
\vdots & \ddots & \vdots & \ddots & \vdots \\
\boldsymbol{\sigma}_{I, 1}^{\prime} & \cdots & \boldsymbol{\sigma}_{I, j}^{\prime} & \cdots & \boldsymbol{\sigma}_{I, J}^{\prime}
\end{array}\right]
$$

In the radar observation, the echo signal is the superposition of all the scattered points. Therefore, it is impossible to segment the echo data in the data domain. The scene segmentation reconstruction method mainly makes use of the characteristics of echo data in different scenes that are unrelated to each other, and ignores the effect of other scene data in the selected sub-scene reconstruction; meanwhile, it will inevitably cause the loss of reconstruction quality. In addition, the observed scene is artificially divided into several sub-scenes during the segmentation operation, and the reconstruction error of the scattered points at the boundary will be increased. In order to make a tradeoff between the reconstruction time and image quality, we can divide the observed scene according to the following principles in the actual operation:

i) Ensure the points of range direction and cross range direction of the segmented sub-scenes are larger than the total measurement data points, which make the sub-scene data occupy a large proportion in the echo data;

ii) In the segmentation of the observed scene, we can appropriately increase the number of segments to the direction which has more discrete points. For example, if the cross range direction has more points, we can appropriately increase the number of segments in the cross range direction, so that the computation complexity will be reduced significantly and the less quality loss of the reconstruction can be ensured, and vice versa;

iii) To adopt the idea of smooth treatment for scene segmentation. That is to say, each sub-scene can overlap with each other, this method can reduce the impact of the data discontinuity at the boundary. In practical, the overlapping points are set as 5-10 to achieve a better reconstruction result.

\section{Performance analysis}

For the above imaging method, the corresponding compressive sensing algorithm must be used. At present, there are many kinds of CS algorithms [9-11] presented, because the orthogonal matching pursuit (OMP) algorithm has the advantages of high reconstruction speed, simple structure, and easy quantitative analysis, it is often used in ISAR imaging [10]. In order to facilitate the comparative analysis, this paper uses the distributed OMP algorithm to dispose the range direction [20], using the OMP algorithm to carry out the cross-range direction processing.

\subsection{Computation complexity analysis}

After the scene segmentation, the sensing matrix is divided into a number of small matrices. The total computation burden is the sum of the computation complexity for the small sub-scenes reconstruction. Specifically, it includes the following aspects:

The traditional CS based reconstruction method needs to stack the column of the data matrix in one column vector. Assuming that $G$ is the sparse degree of the observed scene, thus the total computation cost of the traditional method is $T_{1}=O(G M H N a P Q)$ [16]. For the CS reconstruction method based on the two-dimensional decoupling model, the computation complexity mainly focuses on (6) and (7). Suppose that the sparse degree of the range and cross range are $K_{1}$ and $K_{2}$, respectively. Therefore, the total computation burden is $T_{2}=N a O\left(K_{1} M H P\right)+$ $\mathrm{PO}\left(\mathrm{K}_{2} \mathrm{Na} \mathrm{a}^{\prime} \mathrm{Q}\right)$. For the proposed method, the computation cost mainly focuses on (9) and (14), respectively. Because of the scene segmentation, the sparse degree of the range and cross range for each sub-scene are changed to $K_{1} / I$ and $K_{2} / J$, respectively. Then the total computation complexity is $T_{3}=\mathrm{NaO}\left(K_{1} M H P / I\right)+P O\left(K_{2} N a^{\prime} Q / J\right)$.

It can be seen that the computation cost of the proposed method is much lower than the other two methods. It is obvious that the more segments can contribute to smaller matrices, which results in reduced computation cost. Since each sub-scene is independent of each other, if we take the method of parallel processing, the processing speed will be faster. 


\subsection{Storage space requirement analysis}

The larger the dimension of the sensing matrix is, the more storage space is needed. The sensing matrix dimension of the method 1 equals to the dimension of the data matrix multiplied by the dimension of the reconstructed scene, i.e. $M H N a \times P Q$. The dimension of the sensing matrix required by the range reconstruction of the method 2 is $M H \times P$, and the cross range is $N a^{\prime} \times Q$. For the proposed method, the dimension of the sensing matrix is related to the number of the segments. For the range reconstruction, the dimension of the sensing matrix is $M H \times P / I$. And for the cross range direction, the dimension of the sensing matrix is $N a^{\prime} \times Q / J$.

Comparing the storage space requirements of these three methods, we can see that the storage space required by the proposed method is the lowest. Based on the conditions of the previous hypothesis, the max dimensions of the sensing matrix are $16384 \times 262144$ and $128 \times 512$ for the other two methods, respectively. Assuming the segmentation number is 4 , and the max sensing matrix dimension of the proposed method reduces to $128 \times 128$, which is suitable for the reconstruction of the larger scene and the configuration of the computer is easy to satisfy.

\subsection{Reconstruction accuracy analysis}

In fact, the echo signal is the linear superposition of all the scattered points in the observed scene, which cannot be separated from the data field. For the proposed method, the echo data are not divided too. It is assumed that the information of scattered points in the other sub-scenes has no influence on the reconstruction of the selected sub-scene, and only uses the data related to the sub-scene in the echo. Take the range segmentation as an example, for the selected sub-scene, the echo data $\boldsymbol{S}\left(n_{a}\right)$ can be expressed as

$$
\boldsymbol{S}\left(n_{a}\right)=\boldsymbol{S}_{1}\left(n_{a}\right)+\boldsymbol{S}_{2}\left(n_{a}\right)
$$

where $\boldsymbol{S}_{1}\left(n_{a}\right)$ is the data which belong to the selected subscene, and $\boldsymbol{S}_{2}\left(n_{a}\right)$ denotes the data corresponding to other sub-scenes.

Assuming that the OMP algorithm is used, it will remove the strongest scattered point data from the original echo data and then search for the next scattered point in each loop. The update process for data $\boldsymbol{S}\left(n_{a}\right)$ can be expressed as

$$
\begin{gathered}
\boldsymbol{r}_{k}=\boldsymbol{S}\left(n_{a}\right)-\boldsymbol{\Psi}_{i}^{T^{k}}\left(n_{a}\right) \widehat{\boldsymbol{\theta}}_{i}^{\prime}= \\
\boldsymbol{S}_{1}\left(n_{a}\right)-\boldsymbol{\Psi}_{i}^{T^{k}}\left(n_{a}\right) \widehat{\boldsymbol{\theta}}_{i}^{\prime}+\boldsymbol{S}_{2}\left(n_{a}\right)
\end{gathered}
$$

where $\boldsymbol{T}^{k}$ denotes the support set for the $k$ th loop, $\widehat{\boldsymbol{\theta}}_{i}^{\prime}$ is the value of non-zero elements.
It can be found from (18) that only the selected scene data $\boldsymbol{S}_{1}\left(n_{a}\right)$ are updated in each loop, but the data $\boldsymbol{S}_{2}\left(n_{a}\right)$ always exist, which will affect the reconstruction of the selected sub-scene, resulting in the reduction of the reconstruction precision. This effect is not obvious when the number of segments is small, and the reconstruction loss will be more obvious when the number of segments becomes larger. Therefore, in practice, it can be operated according to the principle of scene segmentation which is proposed in the last section.

\section{Simulation analysis}

\subsection{Performance analysis with simulated data}

To show the performance of the proposed algorithm, simulation is performed. With reference to typical ISAR scenarios, the parameters are selected based on an SF radar system employing RCFS signals, which are listed in Table 1. Thus the total bandwidth is $N \Delta f=500 \mathrm{MHz}$.

Table 1 Radar parameters

\begin{tabular}{cccccc}
\hline Parameter & $f_{0} / \mathrm{GHz}$ & $\Delta f / \mathrm{MHz}$ & $B / \mathrm{MHz}$ & $T_{p} / \mathrm{us}$ & $N$ \\
\hline Value & 10 & 5 & 5 & 4 & 100 \\
\hline \hline Parameter & $\mathrm{PRF} / \mathrm{Hz}$ & $f_{s} / \mathrm{MHz}$ & $\mathrm{SNR} / \mathrm{dB}$ & $N a$ & $M$ \\
\hline Value & 3000 & 0.5 & 5 & 128 & 64 \\
\hline
\end{tabular}

The target model is illustrated in Fig. 2. The intensity of the scattered points is modeled as one-mean Gaussian distributed variables with standard deviation 1 .



Fig. 2 Target model

\section{Simulation 1 Effectiveness verification}

Assuming that the range and cross-range are sampled into two vectors with points of $P=256$ and $Q=192$, respectively. Seventy percent of the data in the cross-range direction are used and the overlapping points are set as five in the range and cross-range directions, respectively. Fig. 3 is the reconstruction result under different numbers of segments when the SNR is $5 \mathrm{~dB}$.

It can be observed from Fig. 3 that with the number of segments increases, the image quality does not change significantly. Only a small number of false reconstruction points appear when the segmentation number is 16 , but 
the image quality is still good. Therefore, the proposed method is valid and feasible.

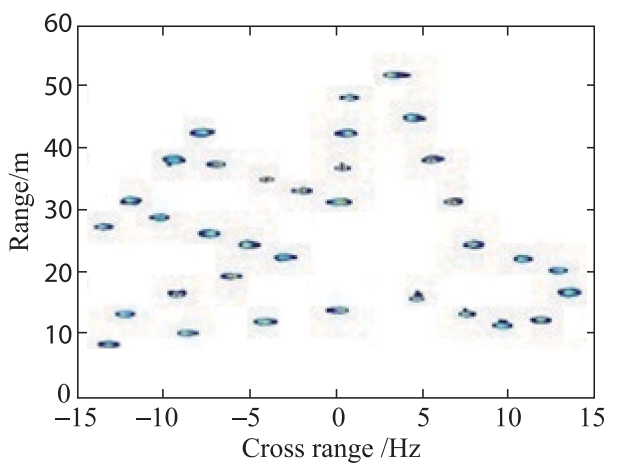

(a) One scenes $(I=1 ; J=1)$

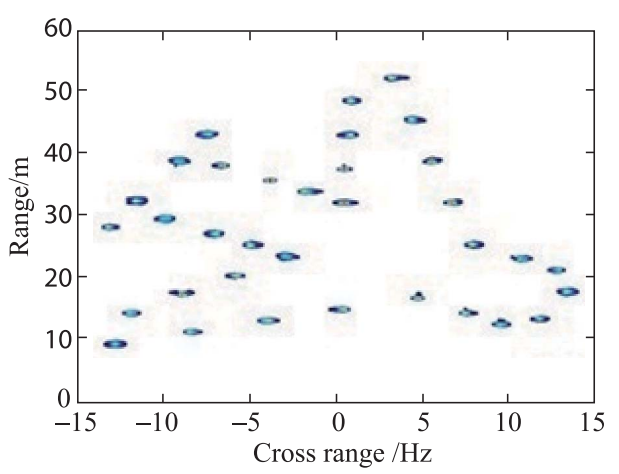

(b) Two sub-scenes $(I=2 ; J=1)$

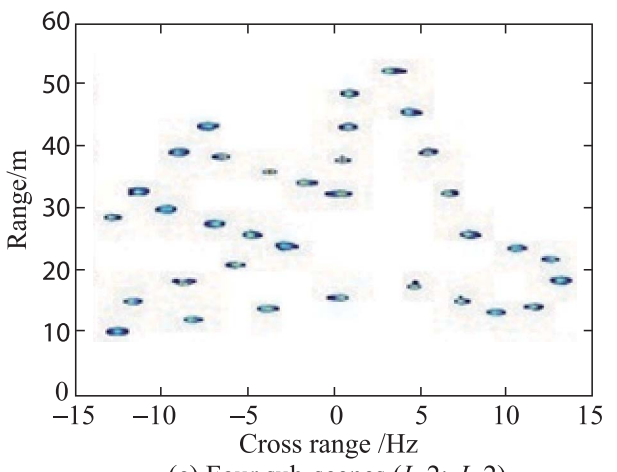

(c) Four sub-scenes $(I=2 ; J=2)$

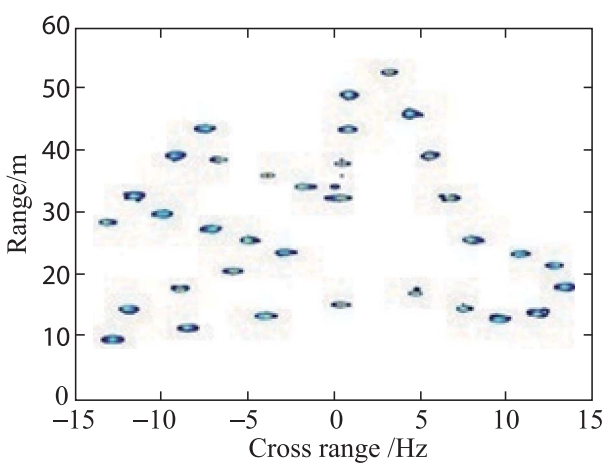

(d) Six sub-scenes $(I=3 ; J=2)$

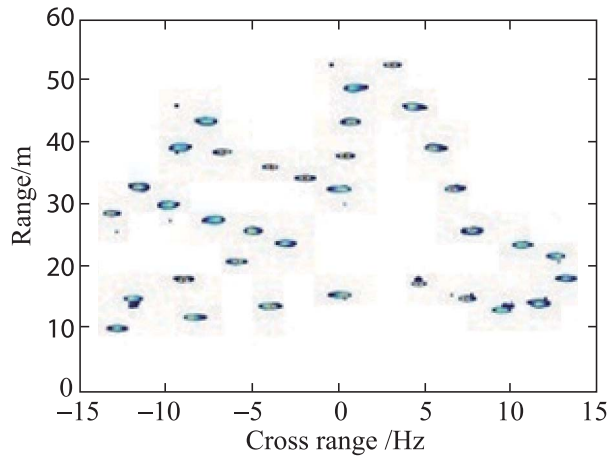

(e) Nine sub-scenes $(I=3 ; J=3)$



(f) Sixteen sub-scenes $(I=4 ; J=4)$

Fig. 3 Reconstruction result under the different number of segments

Simulation 2 Reconstruction time comparison

The following experiments are run on a personal computer with Core $2.93 \mathrm{GHz} \mathrm{CPU}$ and Matlab 7.8.0. The imaging parameters are the same as Simulation 1. Fig. 4 is the reconstruction time required for the proposed method under different numbers of segments.

From Fig. 4, we can find that when the scene is segmented, the reconstruction time is significantly reduced. The reconstruction time decreases more significantly, especially in the case of a large number of segments. If the sub-scenes are processed in parallel, the reconstruction speed will be quicker, and it fully shows the advantages of this proposed method.

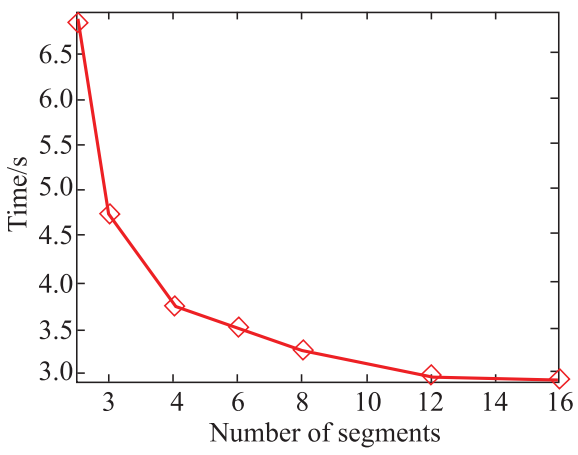

Fig. 4 Reconstruction time required 
Simulation 3 Performance of imaging reconstruction

In order to show the imaging performance of the new method, the imaging quality is measured by entropy and target-to-background ratio (TBR) [21] in this section. If the conditions are unchanged, Fig. 5 and Fig. 6 are the entropy and TBR of the imaging result under different SNR conditions.


Fig. 5 Entropy values

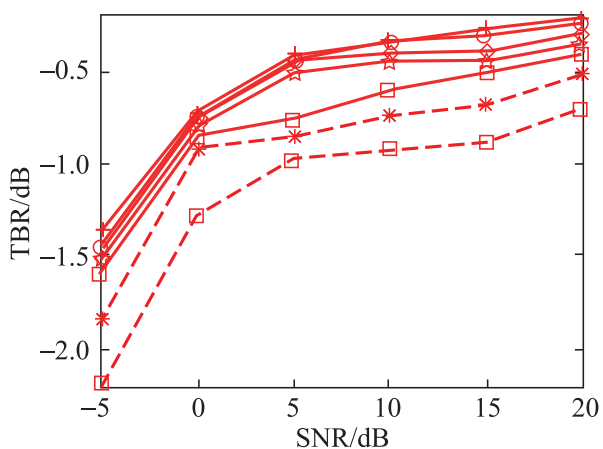

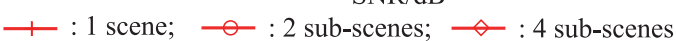
$\neg$ : 6 sub-scenes; $\square: 9$ sub-scenes; - - * - : 12 sub-scenes; 一曰 - : 16 sub-scenes.

Fig. 6 TBR values

By comparing results under different SNRs and numbers of segments, we conclude that the lower the SNR is, the higher entropy and lower TBR values are, which means the image quality decreases. When the number of segments is smaller than six, image entropy and TBR values are approximately unchanged, so is the imaging quality under the same SNR conditions. As the number of segments increases, the proportion of sub-scene data in the total measurement data gradually decreases, and the image quality becomes worse. When the SNR is higher than $5 \mathrm{~dB}$, entropy and TBR values are approximately unchanged, which means that the quality of the imaging result is steady. When the SNR is $-5 \mathrm{~dB}$, the imaging quality decreases dramatically.

\subsection{Performance analysis with measured data}

In order to further validate our method, the measured data are utilized in [22]. The overlapping points are set as five and $80 \%$ of the data in the range and cross range directions are used. The imaging results under different numbers of segments are depicted in Fig. 7.

Visual inspection of these results reveals that the quality of the image is degraded along with the increasing number of segments in accordance with the simulation results showed in Fig. 7. However, when the number of segments is smaller than 16 , the image quality can also be accepted. But when the scene is divided into 16 sub-scenes, the quality of the reconstructed image would be relatively poor and could no longer be accepted. The reconstruction time required and the entropy and TBR of the image for the proposed method are shown in Fig. 8-Fig. 10.

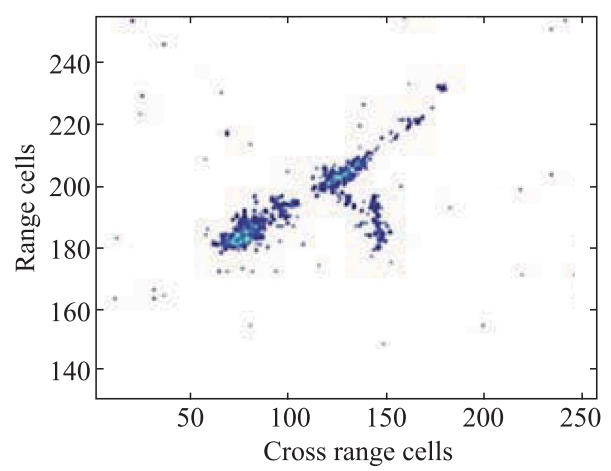

(a) One scenes $(I=1 ; J=1)$



(b) Two sub-scenes $(I=2 ; J=1)$

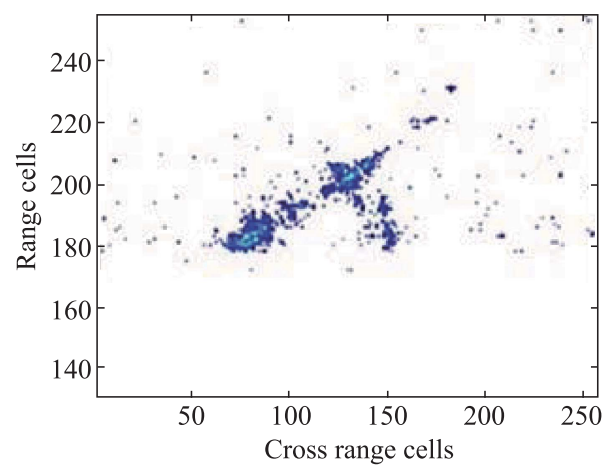

(c) Four sub-scenes $(I=2 ; J=2)$ 




(d) Six sub-scenes $(I=3 ; J=2)$



(e) Nine sub-scenes $(I=3 ; J=3)$



(f) Sixteen sub-scenes $(I=4 ; J=4)$

Fig. 7 Reconstruction results under different numbers of segments

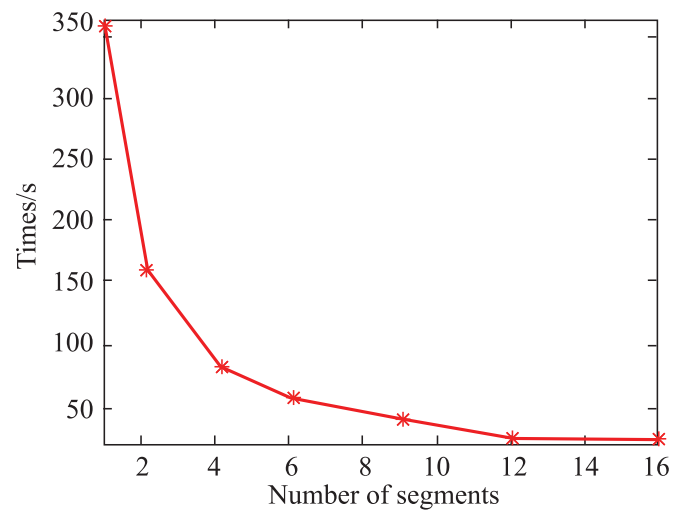

Fig. 8 Reconstruction time required

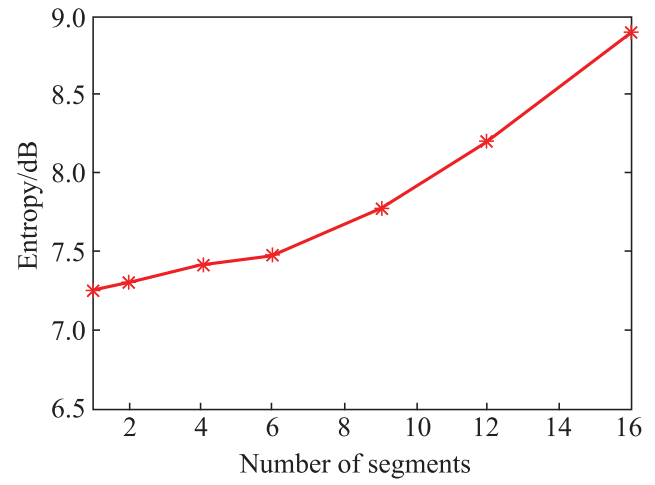

Fig. 9 Entropy values

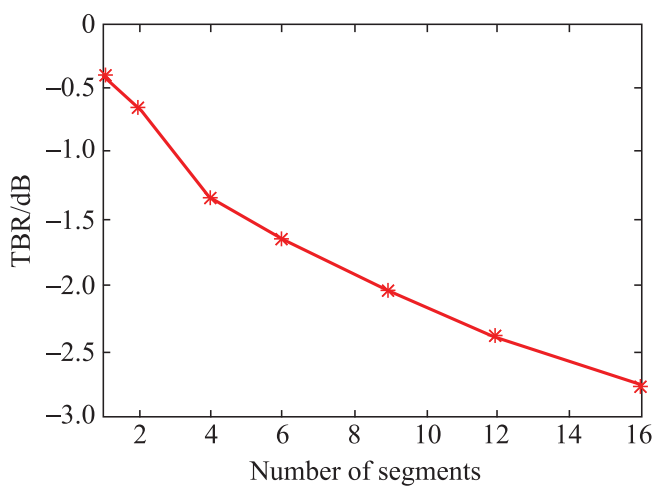

Fig. 10 TBR values

From Fig. 8 - Fig. 10, we can draw the same conclusion as the previous section. Therefore, the results verify the correctness and effectiveness of the new method.

\subsection{Selection criterion}

Because the image quality of the new method is decreased with the increasing of the number of segments, it also limits the further reduction of the reconstruction time. In practical, we need to make a tradeoff between the number of segmentation and the quality of the image. In order to give the reference criterion for the selection of the segmentation number in real applications, this paper defines an entropy and processing time based cost index (CI) to evaluate the performance of the new algorithm under different segmentation number conditions. The cost index is defined as

$$
\mathrm{CI}=\operatorname{abs}\left(\frac{[T(i)-T(1)] / T(1)}{[\operatorname{En}(i)-E n(1)] / E n(1)}\right)
$$

where $T(i)$ denotes the processing time for the $i$ th scene segmentation. Similarly, $\operatorname{En}(i)$ denotes the entropy of the imaging result for the $i$ th scene segmentation.

In (19), the imaging performance is measured under the condition of different segmentation numbers according to the ratio of the entropy change rate and the change rate of the processing time. That is to say, the larger the CI 
value is, the magnitude of the processing time reduction is greater than the magnitude of the image quality reduction. It also shows that the corresponding segmentation number is better. The following Table 2 shows the cost index value under different segmentation numbers.

Table 2 Cost index value under different segmentation numbers

\begin{tabular}{ccc}
\hline \multirow{2}{*}{$\begin{array}{c}\text { Segmentation } \\
\text { number }\end{array}$} & \multicolumn{2}{c}{ CI value } \\
\cline { 2 - 3 } & Simulated data & Measured data \\
\hline 2 & 53.9 & 108.43 \\
4 & 34.03 & 36.98 \\
6 & 35.38 & 27.06 \\
9 & 21.25 & 12.72 \\
12 & 14.59 & 7.18 \\
16 & 11.53 & 4.13 \\
\hline
\end{tabular}

Apparently, when the segmentation number is 2, we can get the largest CI value, which means that the highest cost performance of the proposed method. This index suggests the better selection criterion of the segmentation number in real applications.

\section{Conclusions}

In this paper, a fast imaging method of RCFS ISAR is proposed based on the idea of scene segmentation. By dividing the large scene into several sub-scenes, the reconstruction time and the memory storage requirement is reduced. This method can also reduce the requirement of hardware, and it is suitable for the reconstruction of large observed scenes. It is necessary to note that the segmentation number and the overlapping points affect the image quality, since only segmentation number is considered. We plan to further investigate the corresponding issue in future work.

\section{References}

[1] D. L. Donoho. Compressed sensing. IEEE Trans. on Information Theory, 2006, 52(4): 1289-1306.

[2] S. L. Zhang, F. Xi, S. Y. Chen, et al. Segment-sliding reconstruction of pulsed radar echoes with sub-Nyquist sampling. http://arxiv.org/abs/1503.00434, 2015.

[3] Q. K. Hou, Y. Liu, L. J. Fan, et al. Compressed sensing digital receiver and orthogonal reconstructing algorithm for wideband ISAR radar. SCIENCE CHINA Information Sciences, 2015, 58: 020302.

[4] E. Ertin. Frequency diverse waveforms for compressive radar sensing. Proc. of Waveform Diversity \& Design Conference, 2010: $216-219$.

[5] N. Sugavanam, E. Ertin. Recovery guarantees for multifrequency chirp waveforms in compressed radar sensing. http://120.52.73.76/arxiv.org/pdf/1508.07969v1, 2015.

[6] H. X. Wang, Y. Liang, M. D. Xing, et al. ISAR imaging via sparse frequency-stepped chirp signal. Scientia Sinica (Informationis), 2011, 41(12): 1529-1540. (in Chinese)

[7] F. Zhu, Q. Zhang, Y. Luo, et al. A novel cognitive ISAR imaging method with random stepped frequency chirp signal. SCIENCE CHINA Information Sciences, 2012, 55(8): $1910-$ 1924.
[8] L. Zhang, Z. J. Qiao, M. D. Xing, et al. High-resolution ISAR imaging with sparse stepped-frequency waveforms. IEEE Trans. on Geoscience and Remote Sensing, 2011, 49(11): $4630-4651$.

[9] S. D. Li, J. Yang, W. F. Chen, et al. Overview of radar imaging technique and application based on compressive sensing theory. Journal of Electuonics \& Information Technology, 2016, 38(2): 495-508. (in Chinese)

[10] J. A. Tropp, A. C. Gilbert. Signal recovery from random measurements via orthogonal matching pursuit. IEEE Trans. on Information Theory, 2007, 53(12): 4655-4666.

[11] W. Dai, O. Milenkovic. Subspace pursuit for compressive sensing signal reconstruction. IEEE Trans. on Information Theory, 2009, 55(5): 2230-2249.

[12] G. Aboozar, B. Z. Massoud, J. Christian. Sparse decomposition of two dimensional signals. Proc. of the IEEE International Conference on Acoustics, Speech and Signal Processing, 2009: 3157-3160.

[13] S. Y. Li, G. Q. Zhao, H. M. Li, et al. Near-field radar imaging via compressive sensing. IEEE Trans. on Antennas and Propagation, 2015, 63(2): 828-833.

[14] S. D. Li, W. F. Chen, J. Yang, et al. A fast two dimensional joint linearized bregman iteration algorithm for super-resolution inverse synthetic aperture radar imaging at low signal-to-noise ratios. Acta Physica Sinica, 2016, 65(3): 038401. (in Chinese)

[15] S. D. Li, W. F. Chen, J. Yang, et al. A fast complex linearized Bregman iteration algorithm and its application in ISAR imaging. Scientia Sinica (Informationis), 2015, 49(5): 1179-1196. (in Chinese)

[16] J. G. Yang, J. Thompson, X. T. Huang, et al. Segmented reconstruction for compressed sensing SAR imaging. IEEE Trans. on Geoscience and Remote Sensing, 2013, 51(7): 4214 - 4225.

[17] Z. Bao, M. D. Xing, T. Wang. Radar imaging technology. Beijing: Publishing House of Electronics Industry, 2006. (in Chinese)

[18] L. F. Ding, F. L. Geng, J. C. Chen. Radar principles. 5th ed. Beijing: Publishing House of Electronics Industry, 2014. (in Chinese)

[19] M. F. Duarte, S. Sarvotham, D. Baron, et al. Distributed compressed sensing of jointly sparse signals. Proc. of the 39th Asilomar Conference on Signals, Systems \& Computers, 2005: $1537-1541$.

[20] D. Sundman. Greedy algorithms for distributed compressed sensing. Stockholm, Sweden: KTH Royal Institute of Technology, 2014.

[21] L. Zhang. Study on high resolution SAR/ISAR imaging and error correction. Xi' an: Xidian University, 2012. (in Chinese)

[22] Y. J. Huang, X. Z. Wang, X. L. Li, et al. Inverse synthetic aperture radar imaging using frame theory. IEEE Trans. on Signal Processing, 2012, 60(10): 5191-5200.

\section{Biographies}

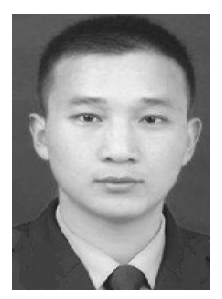

Mingjiu Lü was born in 1985. He received his master degree from the Air Force Early Warning Academy in 2010. He is a Ph.D. candidate at the Air Force Early Warning Academy, Wuhan, China. His research interests are compressed sensing and ISAR imaging.

E-mail: 1v_mingjiu@163.com 


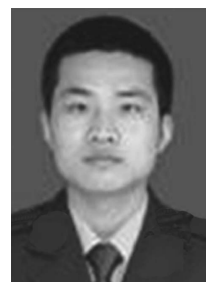

Shaodong Li was born in 1987. He is a Ph.D. at Air Force Early Warning Academy, Wuhan, China. His research interests are compressed sensing and inverse synthetic aperture radar imaging. E-mail: liying198798@126.com

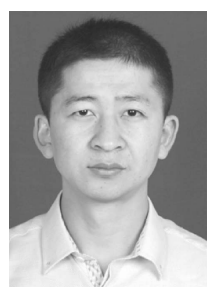

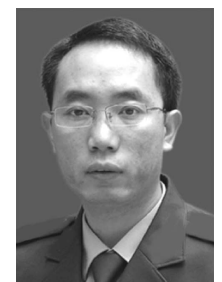

Jun Yang was born in 1973. He received his Ph.D. degree from Air Force Engineering University, Xi'an, China in 2003. Now, he is an associate professor at the Air Force Early Warning Academy, Wuhan, China. His research interests are radar system, radar imaging, and compressed sensing.

E-mail: yangjem@sina.com

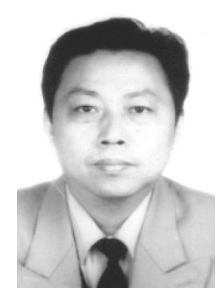

Xiaoyan Ma was born in 1962. He received his Ph.D. degree from Tsinghua University, Beijing, China in 2002. He is currently a full professor and the dean of Air Force Early Warning Academy, Wuhan, China. His main research interests include radar system design, target detection and imaging, as well as ISAR signal processing.

E-mail: mxyldxy@sina.com 